\title{
NORMAS DE EFICIENCIA ENERGÉTICA Y SEGURIDAD ELÉCTRICA
}

Ing. Miguel Maduri (*)

Ouantas veces como usuarios de una instalación luminotécnica, de materiales eléctricos o de productos electrónicos y/o electrodomésticos, ya sean importados y/o nacionales nos preguntamos si los mismos son seguros, si son eficientes. Es decir desconocemos como identificar a simple vista o como, poder saber sobre los mismos, respecto a su eficiencia energética y/o, si no estamos en presencia de un riesgo eléctrico ante su uso.

\section{EFICIENCIA ELECTRICA:}

Nuestro país en el año 1994, mediante la Ley No 24.295, aprobó la Convención Marco de las Naciones Unidas sobre el cambio climático (CMNUCC) y a su vez mediante la Ley No 25.438, en el año 2001, aprobó el Protocolo de KYOTO (PK) de esa Convención.

El cambio climático es debido al "efecto invernadero" producido por los gases asociados al denominado calentamiento de la atmósfera terrestre. Entre ellos está el dióxido de carbono ( $\mathrm{CO} 2)$, que procede mayoritariamente del consumo de combustibles fósiles; el metano (CH4), el óxido nítrico (N2O), y tres gases industriales fluorados empleados como refrigerantes, conductores del calor y aislantes: el carbón hidrofluorico (HFC), el carbón perfluorico (PFC) y el hexafluoruro de azufre (SF6).

El uso eficiente de la energía eléctrica, significa menor producción de $(\mathrm{CO} 2)$ a la atmosfera.

El uso eficiente de la energía eléctrica, significa, consumir menos, minimizando el impacto sobre el ambiente, optimizando la conservación de la energía.El uso eficiente de la energía eléctrica, significa, consumir menos (si bien hay un ahorro económico y energético), significa saber hacerlo, haciendo un uso responsable cada día sin derroche.

Que podemos hacer en lo cotidiano:

A) Por el momento: Consumir haciendo el mejor uso de los recursos, sin auto - prohibirse, ni disminuir los niveles de confort, de producción y/o las necesidades cotidianas de prestación, evitando el derroche, regulando la temperatura de los equipos de aires acondicionados, apagando las luces innecesarias, o los equipos que no se usan como ser PC, TV, Equipo de música, etc. Usando la función apagado y no desde el control remoto, usando la tecla del stand by.

En el caso de los monitores de las PC, cuando no se los usa, los equipos tienen funciones de ahorro, como por ejemplo los

(*) Ing. Electricista, Profesor Adjunto de las materias: Instalaciones Eléctricas y Estaciones Transformadoras en la UNCo- fac. Ing. Neuquen. Dtor del Proyecto de extensión “Uso Seguro y Eficiente de la Electricidad. maduri@neunet. com.ar 
"Energy Star" estos resultan muy efectivos ya que posibilitan disminuir el consumo energético, (pasan al estado de bajo consumo cuando no están siendo usados).

B) Consumir haciendo uso de lámparas, equipos electrodomésticos y/o electrónicos que sean eficientes, es decir elementos y/o aparatos (lámparas, motores, electrodomésticos y/o electrónicos) que a igual prestación y/o servicio, tienen un menor consumo de energía eléctrica.

En el ámbito nacional, se vienen desarrollado en las últimas décadas varias acciones desde el Poder Ejecutivo que están orientadas a lograr un Uso Eficiente de la Energía, no solo en el hogar, sino también en el Alumbrado Público, en la Industria, en el sector de los Edificios Públicos, Transporte, etc. posibilitando a su vez el ahorro energético a nivel país. Entre las acciones implementadas tenemos:

1.- Resolución $319 / 99$ de la - Ex - Secretaria de Industria Comercio y Minería de la Nación (SCI\&MN), dispuso en el año 1999 la Resolución $N^{\circ} 319$ donde se estableció la obligatoriedad del uso de Etiquetas de Eficiencia Energética (EEE) para los artefactos eléctricos de uso doméstico que se comercializan en el país.
INTERPRETACIÓN DE LAS ETIQUETAS

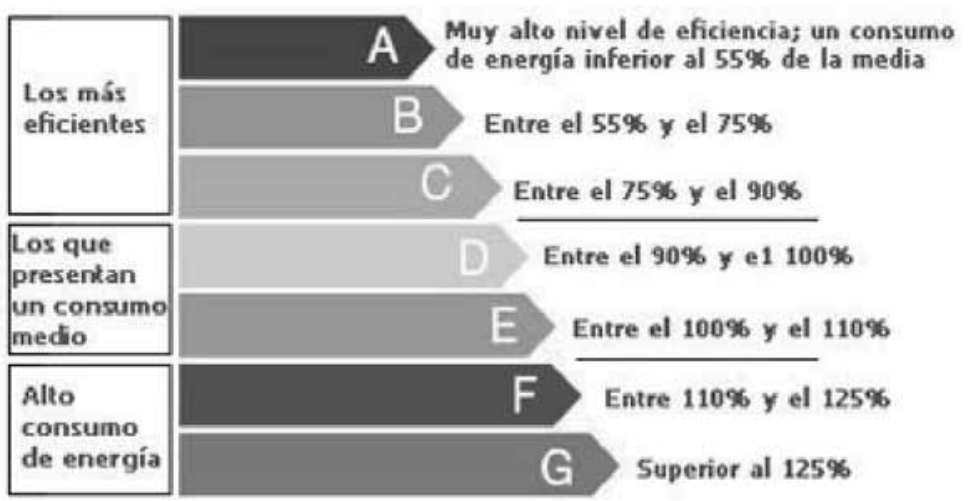

Como se puede ver en el gráfico de barras, existen 7 clases (letras) de eficiencia energética: A, B y C (son las más eficaces en ahorro de energía); D y E (consumo medio); F y G (alto consumo).

\section{2.- Disposición DNCI No 86/07}

Estableció la puesta en vigencia del Etiquetado de Eficiencia Energética obligatorio en lámparas de iluminación general que se comercializan en el país. En lámparas Incandescentes y Fluorescentes compactas, es obligatorio el uso del etiquetado desde noviembre de 2008.

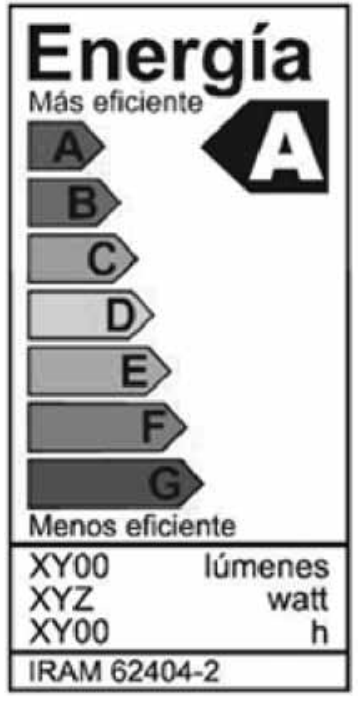

Las etiquetas de las lámparas deben responder a la Norma IRAM 6404 -2.

A la hora de hablar de eficiencia y/o de comparar el consumo energético de una lámpara hay que tener en cuenta ciertos factores:

El flujo lumínico (potencia lumínica que entrega la lampara, se mide en LUMENS). La potencia eléctrica de la lámpara (se mide en WATTS) y la vida nominal del producto (horas promedio de funcionamiento). 


\section{3.- Decreto 140/ 2007 Poder Ejecuti- vo Nacional}

- Declaró de interés y prioridad nacional, el Uso Racional y Eficiente de la Energía (UREE).

- Aprobó los lineamientos del programa Nacional del Uso Racional y Eficiente de la Energía Eléctrica (PRONUREE)

- En el Alumbrado Público - Programa de recambio de todo el alumbrado público ineficiente por iluminación eficiente Medidas de Eficientización consistente en el reemplazo de lámparas incandescentes, mezcladoras, y de mercurio por lámparas de sodio e Instalación de nuevas luminarias $\mathrm{y}$ de atenuadores de potencia.

- Se dispuso el reemplazo de las lámparas Incandescentes por lámparas de bajo consumo en todo el país.

La Ley 26473 estableció, a partir del 31 de diciembre de 2010, la prohibición, importación y comercialización en el país de las lámparas incandescentes.

A su vez, el IRAM también ha acompañado, aprobando e introduciendo nuevas normas respecto a la Eficiencia Energética de: Lámparas, Equipos eléctricos, Electrodomésticos y Electrónicos:

- IRAM 2404 - 3 Aparatos de refrigeración

- IRAM 62404 - 1 Lámparas incandescentes centes

- IRAM 62404 - 2 Lámparas Fluoresdos IRAM 62406 Aires acondiciona-

- IRAM 62405 Motores trifásicos
- IRAM 62407 Balastos para lámparas fluorescentes

- IRAM 2141 - 3 Lavarropas

- IRAM 62408 Bombas centrifugas

- IRAM 62410 Calentadores de agua eléctricos de uso domestico

- IRAM 62411 Recetores de TV modo encendido

- IRAM 62301 Medición de consumo en modo espera stand by

- IRAM 19050- 1 Artefactos de cocción a gas Anafes, hornos

\section{SEGURIDAD ELECTRICA}

Desde el punto de vista del riesgo eléctrico, existen tres aspectos a tener en cuenta:

$\square$ Instalaciones eléctricas defectuosas;

$\checkmark$ El uso no seguro de la electricidad

$\checkmark$ Artefactos eléctricos no seguros (fuera de normas).

El Riesgo eléctrico, se manifiesta como un incidente eléctrico a través de una descarga eléctrica, un cortocircuito, una sobrecarga, un principió de incendio, emanaciones de gases tóxicos, etc. y en el peor de los casos como un accidente (muchas veces aleatorio) provocando la pérdida de un ser querido.

En nuestro país también desde el ámbito nacional, se han desarrollado varias acciones desde el Poder Ejecutivo donde contamos con leyes, normas y disposiciones respecto al tema de la seguridad eléctri$\mathrm{ca}$, que deben cumplirse y ser controladas?. 
Cuáles son las disposiciones constructivas en el tema instalaciones eléctricas que están vigentes y que debernos conocer? A modo informativo se tienen, las siguientes: (para más información, se sugiere ver cada una en particular.)

- La ley No 24240 de Defensa del Consumidor: Establece para los productores, importadores, distribuidores y vendedores, el deber de informar a los consumidores sobre los bienes y servicios que estos adquieren y de proteger su salud. Indicando claramente:

$\checkmark \mathrm{Su}$ denominación

$\checkmark$ Nombre del país donde fueron producidos o fabricados

$\checkmark$ Su calidad, pureza o mezcla (en productos eléctricos esto se demuestra exhibiendo el producto la norma de fabricación IEC ó equivalente IRAM)

$\checkmark$ Las medidas netas de su contenido.

- Ley 19587 Higiene y Seguridad en el Trabajo - Decreto 351

- La Resolución 92/98 de la EX Secretaria de Industria Comercio y Minería de la Nación (SCIMN)

Desde el ámbito privado, también se conocen normas y reglamentaciones, respecto a la seguridad eléctrica, como ser:

- AEA - Asociación Electrotécnica Argentina: Posee numerosas reglamentaciones y/o reglas particulares referidas a la ejecución de instalaciones eléctricas, como ser alguna de ellas son referidas a:

$\checkmark 903640$ - 7 Reglamentación para la Ejecución de Instalaciones Eléctricas en Inmuebles -

$\checkmark 903640$ - 7 - 710 Reglamentación para la Ejecución de Instalaciones Eléctricas en médicos y salas externas-

$\checkmark 903640$ - 7 - 771 Reglamentación para la Ejecución de Instalaciones Eléctricas en

$\checkmark$ Inmuebles Viviendas, Oficinas y Locales (Unitarios).

$\checkmark 91140$ - Protección Contra los Choques Eléctricos - Aspectos Comunes a las Instalaciones y a los Componentes, Materiales y Equipos

$\checkmark 95703$ - Reglamentación para la Ejecución de Instalaciones Eléctricas de Alumbrado Público.

VER listado de reglamentaciones en http://aea.org.ar

- IRAM - Instituto Argentino de Normalización: Posee números Normas (son documentos

técnicos de normalización) sobre las condiciones mínimas que deben cumplir los

materiales, procedimientos de ensayos y/o equipamiento para ser usados.

VER listado de Normas en http:// www.iram.org.ar

Es de aclarar que, las Normas y reglamentaciones en sí, son documentos públicos, obtenido por consenso entre las partes. Las Normas y Reglamentaciones deben ser consultadas y referenciadas por cada uno de nosotros y/o todos que así lo deseen.

\section{RESOLUCION 92}

La mencionada Resolución 92/98 de la EX Secretaria de Industria Comercio y Minería de la Nación dispuso en el año 1998 
la Resolución $\mathrm{N}^{\circ}$ 92, "Sistema de Certificación de Productos de Baja Tensión" la cual comprende al equipamiento eléctrico luminotécnico, electrónico y/o electrodoméstico que se produce, importa, distribuye o comercializa en el país. Ya han pasado más de 15 años desde su publicación y muy poca gente conoce $y / o$ desconoce su significado (por casa como andamos?), Dicha Resolución debería ser difundida en todas las Escuelas Técnicas y de oficios de todo el país para su conocimiento y aplicación.

La resolución 92/98 estableció lo siguiente:

$\square \quad$ Los requisitos esenciales de seguridad (R.E.S.) del equipamiento eléctrico de Baja Tensión.

$\checkmark \quad$ La libre circulación y comercializa- ción en el país de los productos eléctricos que cumplan con los requisitos esenciales de seguridad

$\checkmark \quad$ Fijo la metodología para certificar el cumplimiento de los requisitos de seguridad.

$\square$ Los R.E.S. se consideran plenamente asegurados si se satisfacen las exigencias de seguridad establecidas en las normas IRAM o IEC aplicables, correspondientes al equipamiento eléctrico considerado.

Alcance: Comprende a todo aparato o equipamiento eléctrico o electrónico, destinado a conectarse a una instalación eléctrica o bien, todo material destinado a formar parte de una instalación eléctrica. Tensión nominal hasta 1000 Volts en C.A. o 1500 Volts en C. C.

Marca IRAM de seguridad

Cumple con las normas de seguridad (IEC o IRAM)
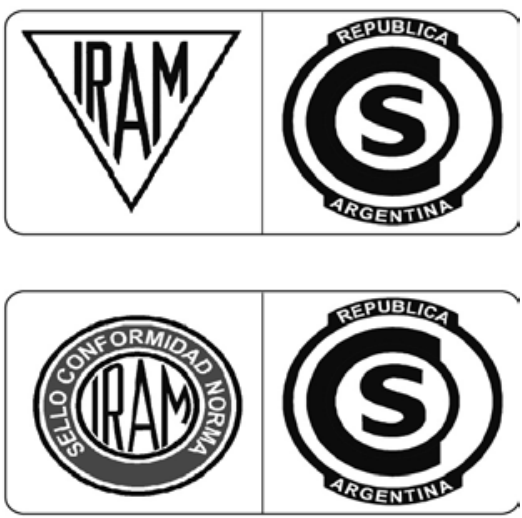

Sello IRAM de Conformidad con Norma IRAM-

Cumple con norma de seguridad y performance

Certificación IRAM de Conformidad de la Fabricación

Cumple con norma IEC de seguridad y performance
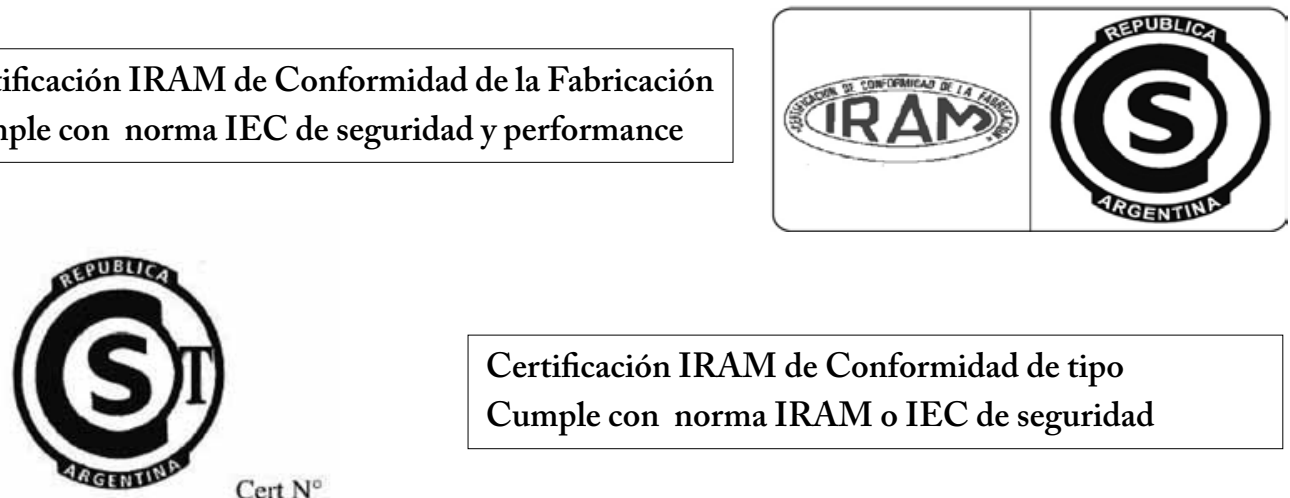

Certificación IRAM de Conformidad de tipo

Cumple con norma IRAM o IEC de seguridad 
El sello debe ir estampado sobre el producto, su envase o etiquetado. El sello no asegura el correcto funcionamiento del producto, solo garantiza que el producto adquirido cumple las normas y por ende es seguro su uso por doña Rosa, don Jose o un idóneo.

Para saber si un producto es seguro, a partir del 19/11/2009, deben estar marca- dos de acuerdo a las exigencias de la Resolución 92/98, es decir contar con el sello de seguridad eléctrica y el sello de la entidad certificadora, indicando el país de fabricación y la norma IEC o equivalente IRAM. Dicho grabado debe estar en forma clara y legible en el producto y no en el envase.

Para más información ver WWW. Resolución 92/98

\section{Referencias:}

- Secretaria de Energía de la Nación - ver: www.energia.gov.ar

- IRAM - ver: www.iram.org.ar

- AEA - ver: www.aea.org.ar

- APSE - ver: www.apse.org.ar

- INFOLEG - ver: www.infoleg.gov.ar

- PRONUREE - ver decreto 140/2007 en INFOLEG 